\title{
On tetrahedrally closed line systems and a generalization of the Haemers-Roos inequality
}

\author{
Bart De Bruyn
}

Ghent University, Department of Mathematics: Algebra and Geometry, Krijgslaan 281 (S25), 9000 Gent (Belgium); Bart.DeBruyn@Ugent.be

\begin{abstract}
We generalize the well-known Haemers-Roos inequality for generalized hexagons of order $(s, t)$ to arbitrary near hexagons $\mathcal{S}$ with an order. The proof is based on the fact that a certain matrix associated with $\mathcal{S}$ is idempotent. The fact that this matrix is idempotent has some consequences for tetrahedrally closed line systems in Euclidean spaces. One of the early theorems relating these line systems with near hexagons is known to contain an essential error. We fix this gap here in the special case for geometries having an order.
\end{abstract}

MSC2010: 05B20, 05B25, 51E12, 52C35

Keywords: line systems, tetrahedrally closed, near polygon, generalized polygon

\section{Introduction}

Consider the $n$-dimensional Euclidean space $\mathbb{R}^{n}$ with origin $o$, and suppose $\mathcal{L}$ is a nonempty set of lines through $o$. If $L_{1}, L_{2} \in \mathcal{L}$, then $\theta_{L_{1}, L_{2}} \in\left[0, \frac{\pi}{2}\right]$ denotes the angle between the lines $L_{1}$ and $L_{2}$. The set $\mathcal{L}$ is called a line system of type $\left(a_{1}, a_{2}, \ldots, a_{k}\right)$, where $0 \leq a_{1}, a_{2}, \ldots, a_{k} \leq 1$, whenever $\cos \left(\theta_{L_{1}, L_{2}}\right) \in\left\{a_{1}, a_{2}, \ldots, a_{k}\right\}$ for any two distinct lines $L_{1}$ and $L_{2}$ of $\mathcal{L}$. Line systems of type $\left(a_{1}, a_{2}, \ldots, a_{k}\right)$ of $\mathbb{R}^{n}$ are equivalent with systems of vectors of type $\left(a_{1}, a_{2}, \ldots, a_{k}\right)$ of $\mathbb{R}^{n}$. Such systems of vectors of $\mathbb{R}^{n}$ are nonempty sets $\Sigma$ of vectors of $\mathbb{R}^{n}$ that satisfy the following:

- $-\bar{v} \in \Sigma$ for every $\bar{v} \in \Sigma$;

- the (squared) norm $\bar{v} \cdot \bar{v}$ of any $\bar{v} \in \Sigma$ is a nonzero constant $c$;

- if $\bar{v}_{1}, \bar{v}_{2} \in \Sigma$ and $\bar{v}_{2} \notin\left\{\bar{v}_{1},-\bar{v}_{1}\right\}$, then $\frac{\left|\bar{v}_{1} \cdot \bar{v}_{2}\right|}{c} \in\left\{a_{1}, a_{2}, \ldots, a_{k}\right\}$.

Line systems of type $\left(a_{1}\right)$ are also known as equiangular line systems. Such line systems were first studied by Haantjes [17] and van Lint \& Seidel [23], and have ever since been 
investigated by several other researchers, see e.g. $[16,28]$ and the references therein. They are also related to other combinatorial objects, like two-graphs [27].

Line systems of type $\left(0, \frac{1}{2}\right)$ were first studied by Cameron et al. [5] and those of type $\left(0, \frac{1}{3}\right)$ by Shult and Yanushka [29], and subsequently by Neumaier [25] and Cuypers [10]. Many interesting examples of such line systems exist. The line system spanned by the root systems of the Lie algebras of type $A_{n}, D_{n}, E_{6}, E_{7}$ or $E_{8}$ are examples of line systems of type $\left(0, \frac{1}{2}\right)$. Constructions of line systems of type $\left(0, \frac{1}{3}\right)$ can be found in [29] and [10], with many of the described examples arising from the well known Leech lattice $[22,8]$.

The angle $\theta \in[0, \pi]$ for which $\cos (\theta)$ equals $-\frac{1}{2}$ (respectively, $-\frac{1}{3}$ ) is the angle subtended by two chords drawn from the barycenter of an equilateral triangle (respectively, tetrahedron) to two of its corner vertices. In [5], the notion of a star-closed line system was introduced, meaning that if two lines inclined at $\frac{\pi}{3}$ are present in the line system, then the unique third line in the plane they generate inclined at $\frac{\pi}{3}$ with both of them is also present. A similar notion was introduced in [29]. A line system (of type $\left(0, \frac{1}{3}\right)$ ) of $\mathbb{R}^{n}$ is said to be tetrahedrally closed whenever it has $0,1,2$ or 4 lines in common with every set of four lines obtained by connecting $o$ with the four corners of some tetrahedron centered at $o$. A system $\Sigma$ of vectors of type $\left(0, \frac{1}{3}\right)$ of $\Sigma$ is called tetrahedrally closed whenever the corresponding line system is tetrahedrally closed. Assuming that all norms of the vectors of $\Sigma$ equal a constant $c>0$, then this is equivalent to the condition that if $\bar{v}_{1}, \bar{v}_{2}, \bar{v}_{3} \in \Sigma$ with $\bar{v}_{1} \cdot \bar{v}_{2}=\bar{v}_{1} \cdot \bar{v}_{3}=\bar{v}_{2} \cdot \bar{v}_{3}=-\frac{c}{3}$, then also $\bar{v}_{1}+\bar{v}_{2}+\bar{v}_{3} \in \Sigma$.

The study of tetrahedrally closed line systems of type $\left(0, \frac{1}{3}\right)$ was initiated in [29]. Besides classification results, Shult and Yanushka showed that these line systems were closely related to star-closed line systems of type $\left(0, \frac{1}{2}, \frac{1}{4}\right)$ and to a certain class of pointline geometries which they called near polygons.

Suppose $\Sigma$ is a tetrahedrally closed system of norm 3 vectors of type $\left(0, \frac{1}{3}\right)$ of $\mathbb{R}^{n}$. Then $\bar{v}_{1} \cdot \bar{v}_{2} \in\{0,1,-1,3,-3\}$ for any two vectors $\bar{v}_{1}, \bar{v}_{2} \in \Sigma$. Let $\bar{v} \in \Sigma$ and put $\mathcal{P}_{\Sigma, \bar{v}}:=\{\bar{u} \in \Sigma \mid \bar{v} \cdot \bar{u}=-1\}$. Let $\mathcal{L}_{\Sigma, \bar{v}}$ denote the set of all triples $\left\{\bar{v}_{1}, \bar{v}_{2}, \bar{v}_{3}\right\} \subseteq \mathcal{P}_{\Sigma, \bar{v}}$ such that $\left\{\bar{v}, \bar{v}_{1}, \bar{v}_{2}, \bar{v}_{3}\right\}$ defines a tetrahedron centered at $o$. We say that $\Sigma$ satisfies Property $\left(P_{\bar{v}}\right)$ if $\mathcal{L}_{\Sigma, \bar{v}} \neq \emptyset$ and for any two vectors $\bar{u}_{1}, \bar{u}_{2} \in \mathcal{P}_{\Sigma, \bar{v}}$ with $\bar{u}_{1} \cdot \bar{u}_{2}=1$ there exists a vector $\bar{w} \in \mathcal{P}_{\Sigma, \bar{v}}$ such that $\bar{u}_{1} \cdot \bar{w}=\bar{u}_{2} \cdot \bar{w}=-1$.

Proposition 1.1 ([29, Proposition 3.10]) If $\Sigma$ satisfies Property $\left(P_{\bar{v}}\right)$ for some $\bar{v} \in \Sigma$, then the point-line geometry $\mathcal{S}_{\Sigma, \bar{v}}$ with point set $\mathcal{P}_{\Sigma, \bar{v}}$ and line set $\mathcal{L}_{\Sigma, \bar{v}}$ satisfies the following property:

$(N P)$ for every point $p$ and every line $L$, there exists a unique point on $L$ that is nearest to $p$ (with respect to the distance in the collinearity graph).

Moreover, the diameter of $\mathcal{S}_{\Sigma, \bar{v}}$ is either 1,2 or 3 , and every line of $\mathcal{S}_{\Sigma, \bar{v}}$ is incident with precisely three points. Two points $\bar{w}_{1}$ and $\bar{w}_{2}$ of $\mathcal{S}_{\Sigma, \bar{v}}$ lie at distance $0,1,2$ or 3 from each other depending on whether $\bar{w}_{1} \cdot \bar{w}_{2}$ is equal to $3,-1,1$ or 0 .

A point-line geometry of diameter $d$ satisfying Property (NP) is called a near $2 d$-gon or a near polygon if the value of $d$ is not specified. A near polygon is said to have order 
$(s, t)$ if every line is incident with precisely $s+1$ points and if every point is incident with precisely $t+1$ lines. Near polygons are related to the generalized polygons of Tits [30], to the polar spaces of Tits and Veldkamp [31, 33], to distance-regular graphs [3] and to finite simple groups. While dual polar spaces [4] and generalized polygons (which can be seen as special classes of near polygons) are related to classical groups and exceptional groups of Lie type, there are also several near polygons corresponding to sporadic simple groups, like the $U_{4}(3)$ or Aschbacher near hexagon [1], the Hall-Janko or Cohen-Tits near octagon $[6,7]$ and the $G_{2}(4)$ near octagon [2].

The point-line geometry $\mathcal{S}_{\bar{v}}$ defined in Proposition 1.1 is called a local near polygon of $\Sigma$. In [29, Proposition 3.14], Shult and Yanushka proved that every near $2 k$-gon, $k \in\{1,2,3\}$, with three points per line occurs as a local near polygon of some tetrahedrally closed system of norm 3 vectors of type $\left(0, \frac{1}{3}\right)$. However, soon after their paper was published, it turned out that their arguments contained an essential error. The nature of this error is explained by Neumaier [25, p. 566] who also observed that the result remains valid for those near hexagons for which the collinearity graph is distance-regular (there are nine such geometries). In the present paper we will fill this gap in the original proof for those near hexagons that have an order:

Theorem 1.2 Let $\mathcal{S}$ be a finite near $2 k$-gon of order $(2, t)$ with $k \in\{1,2,3\}$. Then $\mathcal{S}$ occurs as a local near polygon of some tetrahedrally closed system of norm 3 vectors of type $\left(0, \frac{1}{3}\right)$.

In Section 3 we will be able to compute certain intersection numbers in a finite near hexagon $\mathcal{S}$ of order $(s, t)$. This information will be used in Section 4 where we will show that a certain matrix associated with $\mathcal{S}$ is idempotent and thus also positive semidefinite. In Section 6, Theorem 1.2 will be proved from the fact that this matrix is positive semidefinite.

The fact that the matrix is positive semidefinite has additional consequences. It allows to improve a result on generalized polygons obtained by Haemers and Roos [19]. Tits [30] showed that for every prime power $q$ there exists a generalized hexagon of order $(s, t)=\left(q, q^{3}\right)$, the so-called dual twisted triality hexagon associated with the simple group ${ }^{3} D_{4}\left(q^{3}\right)$. These dual twisted triality hexagons are extremal in the sense that $t$ attains its maximal possible value for given $s$. Indeed, Haemers and Roos [19] proved that the inequality $t \leq s^{3}$ holds in any generalized hexagon of order $(s, t)$ with $s>1$. The question whether the dual twisted triality hexagons are the only generalized hexagons of order $\left(s, s^{3}\right), s>1$, remains as of today one of the most important problems in the theory of generalized polygons. In Section 5, we generalize the Haemers-Roos bound for generalized hexagons to arbitrary near hexagons with an order, by proving the following:

Theorem 1.3 Let $\mathcal{S}$ be a finite near hexagon of order $(s, t)$ with $s \neq 1$. Let $x$ and $y$ be two opposite points of $\mathcal{S}$. Let $N$ denote the number of geodesics connecting $x$ and $y$, and put $\overline{t_{2}}:=\frac{N}{t+1}-1$. Then

$$
\overline{t_{2}} \cdot\left(s^{2}+s+1\right)-s^{3} \leq t \leq s^{3}+\overline{t_{2}} \cdot\left(s^{2}-s+1\right) .
$$


If $\mathcal{S}$ is a generalized hexagon, then $\overline{t_{2}}=0$ for any choice of the points $x$ and $y$, and in this case, Theorem 1.3 reduces to the Haemers-Roos inequality $t \leq s^{3}$. In case $\mathcal{S}$ is a regular near hexagon, i.e. a finite near hexagon with an order $(s, t)$ such that any two points at distance 2 have precisely $t_{2}+1$ common neighbours for a certain constant $t_{2}$, then $\overline{t_{2}}$ equals $t_{2}$ for any choice of the points $x$ and $y$. In this case, the upper bound in Theorem 1.3 becomes $t \leq s^{3}+t_{2}\left(s^{2}-s+1\right)$. This upper bound is known as the Mathon or Haemers-Mathon bound. It can be derived using the theory of distance-regular graphs, see $[18,24,26]$. This theory of distance-regular graphs can no longer be used here as the collinearity graph of the near hexagon is not necessarily distance-regular. However, besides geometric and counting arguments, the proof of the present paper still relies on methods from algebraic combinatorics.

For $s>1$, we will see that $\overline{t_{2}} \leq s^{2}$. Taking into account Theorem 1.3, we thus find that $t \leq s^{4}+s^{2}$. So, for any given $s>1$ there can only be a finite number of finite near hexagons of order $(s, *)$. In Section 5 , we will also be able to treat the case where $t$ attains the upper bound $s^{4}+s^{2}$. We will prove the following.

Theorem 1.4 Let $\mathcal{S}$ be a finite near hexagon of order $(s, t)$ with $s \neq 1$. Then $t \leq s^{4}+s^{2}$, with equality if and only if $\mathcal{S}$ is a Hermitian dual polar space of rank 3.

In particular, Theorem 1.4 says that finite near hexagons of order $(2, t)$ can only exist for $t \leq 20$. This improves the upper bound $t \leq 33$ which was achieved in [12, page 120] by means of other techniques.

Previously, the use of algebraic combinatorial tools to study near hexagons was entirely restricted to those that had a distance-regular collinearity graph [3]. The results of the present paper in combination with those of [13] show that the mere fact that the geometries have an order is already sufficient to study them with algebraic combinatorial tools, sometimes leading to surprising results. These algebraic combinatorial methods have led to some breakthrough results where for instance the nonexistence of certain near hexagons was shown whose existence had been open for more than 15 years [11], with no progress being made in the mean time, see [13, Corollary 1]. The nice thing is also that Theorem 1.3 along with a divisibility condition in [13, Theorem 1] immediately kill several near hexagons whose nonexistence proofs in the early literature on the topic needed separate and ad hoc methods.

Theorem 1.3 of the present paper (and [13, Theorem 1.1]) not only have consequences for tetrahedrally closed line systems in Euclidean spaces, but also to the classification of certain nice sets of points in projective spaces, namely to caps $\mathcal{K}$ with the property that every tangent line contains a unique point outside $\mathcal{K}$ that is in at least one secant line. The most notable example of such a nice set is the so-called Coxeter cap in PG $(5,3)$, discovered in [9], on which the Witt design $S(5,6,12)$ can be constructed and whose stabilizer in the full automorphism group of $\mathrm{PG}(5,3)$ is the Mathieu group $M_{12}$. The complete classification of such sets of points (which all have an associated near hexagon with an order) is currently under way by the author and one of its collaborators [14]. 


\section{Preliminaries}

In the present paper, we will always consider a point-line geometry as a pair $\mathcal{S}=(\mathcal{P}, \mathcal{L})$, where $\mathcal{P}$ is the nonempty point set and the line set $\mathcal{L}$ is a collection of subsets of $\mathcal{P}$. If $x$ and $y$ are two points of $\mathcal{S}$, then $\mathrm{d}(x, y)$ denotes the distance between $x$ and $y$ in the collinearity graph, that is the graph whose vertices are the points of $\mathcal{S}$ with two distinct points being adjacent whenever they are incident with the same line. If $x$ is a point and $Y$ a nonempty set of points of $\mathcal{S}$, then we define $\mathrm{d}(x, Y):=\min \{\mathrm{d}(x, y) \mid y \in Y\}$. If $x$ is a point of $\mathcal{S}$ and $i \in \mathbb{N}$, then $\Gamma_{i}(x)$ denotes the set of points at distance $i$ from $x$.

Recall that a point-line geometry with finite diameter $d$ is called a near $2 d$-gon if for every point $x$ and every line $L$ of $\mathcal{S}$, there exists a unique point on $L$ nearest to $x$. A near 0 -gon in this context is just a point (no lines) and a near 2-gon is a line. The near quadrangles are precisely the generalized quadrangles and the degenerate generalized quadrangles (consisting of a number of lines through a point). More generally, a generalized $2 d$-gon is a near $2 d$-gon that satisfies the following additional properties:

(GP1) every point is incident with at least two lines;

(GP2) if $x$ and $y$ are two points of $\mathcal{S}$ at distance $i \in\{1,2, \ldots, d-1\}$ from each other, then there exists a unique neighbour of $y$ at distance $i-1$ from $x$.

The near polygons with only lines of size 2 are precisely the bipartite graphs of finite diameter.

Suppose $\mathcal{S}$ is a near polygon. A quad of $\mathcal{S}$ is a set $Q$ of points satisfying the following:

- if a line of $\mathcal{S}$ has two points in $Q$, then all its points are in $Q$;

- $Q$ is convex, meaning that every point on a shortest path (or geodesic) between two points of $Q$ is also contained in $Q$;

- the subgeometry of $\mathcal{S}$ determined by those points and lines of $\mathcal{S}$ that are contained in $Q$ is a (nondegenerate) generalized quadrangle.

If every line of $\mathcal{S}$ is incident with at least three points, then there exists a constant $t_{Q}$ such that every point of $Q$ is incident with precisely $t_{Q}+1$ lines of $Q$. The following two propositions are taken from Shult and Yanushka [29].

Proposition 2.1 ([29, Proposition 2.5]) Suppose $x_{1}$ and $x_{2}$ are two points at distance 2 in a near polygon $\mathcal{S}$ and $y_{1}$ and $y_{2}$ are two distinct common neighbours of $x_{1}$ and $x_{2}$. If at least one of the lines $x_{1} y_{1}, y_{1} x_{2}, x_{2} y_{2}, y_{2} x_{2}$ contains at least three points, then $x_{1}$ and $x_{2}$ are contained in a unique quad.

Proposition 2.2 ([29, Proposition 2.6]) Suppose $\mathcal{S}$ is a near polygon having at least three points on each line. Then precisely one of the following two cases occurs for a point-quad pair $(p, Q)$ : 
(a) There exists a unique point $x^{\prime}$ in $Q$ nearest to $x$. In this case, $d(x, y)=d\left(x, x^{\prime}\right)+$ $d\left(x^{\prime}, y\right)$ for any point $y \in Q$.

(b) The points in $Q$ nearest to $x$ from an ovoid, i.e. a set of points of $Q$ intersecting each line of $Q$ in a singleton.

If case (a) of Proposition 2.2 occurs, then $x$ is called classical with respect to $Q$. If case (b) occurs, then $x$ is called ovoidal with respect to $Q$.

Proposition 2.3 Suppose $\mathcal{S}=(\mathcal{P}, \mathcal{L})$ is a finite near hexagon of order $(s, t)$ having $v$ points. Put

$$
n_{0}:=1, n_{1}:=s(t+1), n_{2}:=\frac{v}{s+1}-1+s^{2} t-s t, n_{3}:=\frac{s v}{s+1}-s-s^{2} t .
$$

Then $\left|\Gamma_{i}(x)\right|=n_{i}$ for every point $x$ and every $i \in\{0,1,2,3\}$.

Proof. For every point $x$ of $\mathcal{S}$ and every $i \in\{0,1,2,3\}$, we define $n_{i}(x):=\left|\Gamma_{i}(x)\right|$. Since every line $L$ of $\mathcal{S}$ contains a unique point nearest to $x$, the sum $\sum_{y \in L}\left(-\frac{1}{s}\right)^{\mathrm{d}(x, y)}$ is equal to 0. So, $0=\sum_{L \in \mathcal{L}} \sum_{y \in L}\left(-\frac{1}{s}\right)^{\mathrm{d}(x, y)}=\sum_{y \in \mathcal{P}} \sum_{L \ni y}\left(-\frac{1}{s}\right)^{\mathrm{d}(x, y)}=(t+1) \cdot \sum_{y \in \mathcal{P}}\left(-\frac{1}{s}\right)^{\mathrm{d}(x, y)}$, implying that $n_{0}(x)-\frac{n_{1}(x)}{s}+\frac{n_{2}(x)}{s^{2}}-\frac{n_{3}(x)}{s^{3}}=0$. Since $n_{0}(x)=1, n_{1}(x)=s(t+1)$ and $n_{0}(x)+n_{1}(x)+n_{2}(x)+n_{3}(x)=v$, we derive that $n_{i}(x)=n_{i}$ for every $i \in\{0,1,2,3\}$.

Proposition 2.4 Suppose $\mathcal{S}$ is a finite near hexagon of order $(s, t)$ with $s>1$ and $x$ is a point of $\mathcal{S}$. If $\mathcal{R}$ denote the set of quads through $x$, then

$$
\sum_{R \in \mathcal{R}} t_{R}^{2}=t(t+1)-\frac{n_{2}}{s^{2}}
$$

where $n_{2}$ is the constant mentioned in Proposition 2.3.

Proof. If $R \in \mathcal{R}$, then $\left|\Gamma_{2}(x) \cap R\right|=s^{2} t_{R}$, and so the number of points of $\Gamma_{2}(x)$ that are contained in a quad together with $x$ is equal to $\sum_{R \in \mathcal{R}} s^{2} t_{R}$, and the number of other points of $\Gamma_{2}(x)$ is equal to $n_{2}-\sum_{R \in \mathcal{R}} s^{2} t_{R}$. Now, counting in two different ways the number of connections between $\Gamma_{1}(x)$ and $\Gamma_{2}(x)$, we find

$$
s(t+1) \cdot s t=\sum_{R \in \mathcal{R}} s^{2} t_{R}\left(t_{R}+1\right)+n_{2}-\sum_{R \in \mathcal{R}} s^{2} t_{R}
$$

from which it readily follows that $\sum_{R \in \mathcal{R}} t_{R}^{2}=t(t+1)-\frac{n_{2}}{s^{2}}$.

\section{Calculation of some intersection numbers}

In this section, we suppose that $\mathcal{S}$ is a finite near hexagon of order $(s, t)$ having $v$ points. By Proposition 2.3, there exist constants $n_{i}, i \in\{0,1,2,3\}$, such that $n_{i}=\left|\Gamma_{i}(x)\right|$ for every point $x$ of $\mathcal{S}$ and every $i \in\{0,1,2,3\}$. These constants are equal to

$$
n_{0}=1, n_{1}=s(t+1), n_{2}=\frac{v}{s+1}-1+s^{2} t-s t, n_{3}=\frac{s v}{s+1}-s-s^{2} t .
$$


Suppose $x$ and $y$ are two points of $\mathcal{S}$ at distance $\delta$ from each other. For all $i, j \in\{0,1,2,3\}$, let $a_{i j}$ be the intersection number $\left|\Gamma_{i}(x) \cap \Gamma_{j}(y)\right|$. We determine here all $a_{i j}$ 's for $0 \leq i, j \leq$ 2. We deal separately with the various possibilities for $\delta \in\{0,1,2,3\}$.

First, suppose $\delta=0$. Then $a_{i j}=n_{i} \cdot \delta_{i j}$ for all $i, j \in\{0,1,2\}$, where $\delta_{i j}$ denotes the Kronecker delta.

Suppose $\delta=1$. By the triangle inequality, we have $a_{00}=a_{02}=a_{20}=0$. Since $\Gamma_{1}(x) \cap \Gamma_{0}(y)=\{y\}$ and $\Gamma_{0}(x) \cap \Gamma_{1}(y)=\{x\}$, we have $a_{01}=a_{10}=1$. By (NP), the points at distance 1 from $x$ and $y$ are precisely the points of the line $x y$ distinct from $x$ and $y$ implying that $a_{11}=s-1$. By (NP), the points of $\Gamma_{1}(x) \cap \Gamma_{2}(y)$ are precisely the points collinear with $x$ not contained on the line $x y$, showing that $a_{12}=s t$. By symmetry, also $a_{21}=s t$. By $(\mathrm{NP})$, the points of $\Gamma_{2}(x) \cap \Gamma_{2}(y)$ are precisely the points not on the line $x y$ that are collinear with one of the $s-1$ points of $x y \backslash\{x, y\}$, implying that $a_{22}=(s-1) s t$.

Suppose $\delta=2$, and put $a_{11}:=\left|\Gamma_{1}(x) \cap \Gamma_{1}(y)\right|=t_{2}+1$. Note that $t_{2}$ need not to be constant and can depend on the choice of the points $x$ and $y$. Obviously, $a_{02}=a_{20}=1$ and by the triangle inequality we have $a_{00}=a_{10}=a_{01}=0$. If $z \in \Gamma_{1}(x) \cap \Gamma_{2}(y)$, then by $(\mathrm{NP})$, the line $x z$ contains a point collinear with $y$, implying that $x z$ is one of the $t_{2}+1$ lines through $x$ meeting $\Gamma_{1}(y)$. Conversely, each line through $x$ meeting $\Gamma_{1}(y)$ contains $s-1$ points of $\Gamma_{1}(x) \cap \Gamma_{2}(y)$. It follows that $a_{12}=(s-1)\left(t_{2}+1\right)$. By symmetry, also $a_{21}=(s-1)\left(t_{2}+1\right)$. We will now compute $a_{22}$.

If $s=1$, then $\mathcal{S}$ is a bipartite graph implying that $\Gamma_{2}(x) \cap \Gamma_{2}(y)=P \backslash\{x, y\}$, where $P$ is the part of the bipartite graph containing $x$ and $y$. In this case $\mathcal{S}$ has order $(1, t)$, and so both parts of $\mathcal{S}$ have equal size, implying that $a_{22}=\left|\Gamma_{2}(x) \cap \Gamma_{2}(y)\right|=\frac{v}{2}-2$. Assume therefore that $s \geq 2$.

If $t_{2} \geq 1$, then $x$ and $y$ are contained in a unique quad $Q$ by Proposition 2.1. If $t_{2}=0$, then $x$ and $y$ have a unique neighbour $u$, and we define $Q:=x u \cup u y$. Note that in the latter case, $Q$ is convex as well and defines a degenerate generalized quadrangle. Let $\mathcal{R}_{1}$ denote the set of all quads through $x$ meeting $Q$ in a line and let $\mathcal{R}_{2}$ denote the set of all quads through $x$ meeting $Q$ in the singleton $\{x\}$. Then $\mathcal{R}:=\mathcal{R}_{1} \cup \mathcal{R}_{2}$ is the set of all quads through $x$ distinct from $Q$. Regardless of whether $t_{2}=0$ or $t_{2} \geq 1$, we always have

$$
\sum_{R \in \mathcal{R}} t_{R}^{2}=t(t+1)-t_{2}^{2}-\frac{n_{2}}{s^{2}}
$$

by Proposition 2.4. We denote by $N_{1}$ the number of points in $\Gamma_{2}(x) \cap \Gamma_{2}(y) \cap Q$ and by $N_{2}$ the number of pairs $(u, z)$, where $z \in\left(\Gamma_{2}(x) \cap \Gamma_{2}(y)\right) \backslash Q$ and $u \in \Gamma_{1}(x) \cap \Gamma_{1}(z)$.

Lemma 3.1 We have

$$
N_{1}= \begin{cases}s^{2} t_{2}-s t_{2}-s+t_{2} & \text { if } t_{2} \geq 1, \\ 0 & \text { if } t_{2}=0 .\end{cases}
$$

Proof. If $t_{2}=0$, then obviously $N_{1}=0$. If $t_{2} \geq 1$, then $Q$ is a generalized quadrangle of order $\left(s, t_{2}\right)$ containing $(s+1)\left(s t_{2}+1\right)$ points and hence $N_{1}=(s+1)\left(s t_{2}+1\right)-2-$ $2 s\left(t_{2}+1\right)+t_{2}+1=s^{2} t_{2}-s t_{2}-s+t_{2}$. 
Lemma 3.2 We have

$$
N_{2}= \begin{cases}s\left(t-t_{2}\right) t+\left(t_{2}+1\right) s\left(t-t_{2}\right) & \text { if } t_{2} \geq 1 \\ s t^{2}+s t-s & \text { if } t_{2}=0 .\end{cases}
$$

Proof. We count the pairs $(u, z)$, where $z \in\left(\Gamma_{2}(x) \cap \Gamma_{2}(y)\right) \backslash Q$ and $u \in \Gamma_{1}(x) \cap \Gamma_{1}(z)$.

If $u \in \Gamma_{1}(x) \cap \Gamma_{2}(y)$, then by (NP) the line $x u$ contains a unique point collinear with $y$, implying that $u \in Q$. If $u$ has been chosen in such a way and $z \in \Gamma_{1}(u) \cap \Gamma_{2}(y)$, then $u z$ contains a point collinear with $y$, implying that $z \in Q$ since it is on a shortest path between $u \in Q$ and $y \in Q$. So, there are no such pairs $(u, z)$ with $u \in \Gamma_{1}(x) \cap \Gamma_{2}(y)$.

For each of the $s\left(t-t_{2}\right)$ points $u$ of $\Gamma_{1}(x) \cap \Gamma_{3}(y)$, there are $t$ possibilities for $z$, namely one on each line through $u$ distinct from $u x$. Note that if $u$ and $z$ have been chosen in the above way, then $z \notin Q$. Indeed, if $z$ would belong to $Q$, then as $Q$ is convex, we would also have $u \in Q$, which is impossible as $y \in Q$ and $\mathrm{d}(u, y)=3$.

For each of the $t_{2}+1$ points $u$ of $\Gamma_{1}(x) \cap \Gamma_{1}(y)$, there are $s\left(t-t_{2}\right)$ possibilities for $z$ if $t_{2} \geq 1$ and $s(t-1)$ possibilities if $t_{2}=0$. Indeed, the number of lines through $u$ not contained in $Q$ is equal to $t-t_{2}$ (if $t_{2} \geq 1$ ) or $t-1$ (if $t_{2}=0$ ), and on each such line, there are $s$ points in $\left(\Gamma_{2}(x) \cap \Gamma_{2}(y)\right) \backslash Q$. So, the total number $N_{2}$ of pairs is equal to $s\left(t-t_{2}\right) t+\left(t_{2}+1\right) s\left(t-t_{2}\right)$ if $t_{2} \geq 1$ and equal to $s\left(t-t_{2}\right) t+\left(t_{2}+1\right) s(t-1)=s t^{2}+s t-s$ if $t_{2}=0$.

The following is a consequence of Lemmas 3.1 and 3.2.

Corollary 3.3 Regardless of whether $t_{2}=0$ or $t_{2} \geq 1$, we have

$$
N_{1}+N_{2}=s^{2} t_{2}-s t_{2}-s+t_{2}+s\left(t-t_{2}\right) t+\left(t_{2}+1\right) s\left(t-t_{2}\right) .
$$

Lemma 3.4 We have

$$
N_{2}=\sum_{R \in \mathcal{R}} s t_{R}^{2}+a_{22}-N_{1}
$$

Proof. We count the pairs $(u, z)$, where $z \in\left(\Gamma_{2}(x) \cap \Gamma_{2}(y)\right) \backslash Q$ and $u \in \Gamma_{1}(x) \cap \Gamma_{1}(z)$, in another way. If $R \in \mathcal{R}_{1}$, then $R$ contains a unique point $y^{\prime}$ collinear with $y$ and so $y$ is classical with respect to $Q$. Since $\mathrm{d}(x, y)=2$, the point $y^{\prime}$ is also collinear with $x$. In $R$, there are precisely $s t_{R}$ points of $\left(\Gamma_{2}(x) \cap \Gamma_{2}(y)\right) \backslash Q$, namely the $s t_{R}$ neighbours of $y^{\prime}$ in $R$ that are not contained on the line $x y^{\prime}$. If $R \in \mathcal{R}_{2}$, then $R$ does not contain a point of $\Gamma_{1}(y)$, implying that $y$ is ovoidal with respect to $R$, i.e. $\Gamma_{2}(y) \cap R$ is an ovoid of $R$ containing $s t_{R}+1$ points. In $R$, there are then precisely $s t_{R}$ points of $\left(\Gamma_{2}(x) \cap \Gamma_{2}(y)\right) \backslash Q$, namely the $s t_{R}$ points of $\left(\Gamma_{2}(y) \cap R\right) \backslash\{x\}$. We thus see that among the $a_{22}-N_{1}$ points of $\left(\Gamma_{2}(x) \cap \Gamma_{2}(y)\right) \backslash Q$, there are $\sum_{R \in \mathcal{R}} s t_{R}$ points $z_{1}$ that are contained in a quad together with $x$ and $a_{22}-N_{1}-\sum_{R \in \mathcal{R}} s t_{R}$ points $z_{2}$ that are not contained in a quad together with $x$. For each such $z_{1} \in R \in \mathcal{R}$, there are $t_{R}+1$ possibilities for $u$ and for each such $z_{2}$ there is a unique possibility for $u$. Hence, the total number of pairs is also equal to $N_{2}=\sum_{R \in \mathcal{R}} s t_{R}\left(t_{R}+1\right)+a_{22}-N_{1}-\sum_{R \in \mathcal{R}} s t_{R}=\sum_{R \in \mathcal{R}} s t_{R}^{2}+a_{22}-N_{1}$.

By Lemma 3.4, we thus have

$$
a_{22}=N_{1}+N_{2}-\sum_{R \in \mathcal{R}} s t_{R}^{2}
$$


Taking into account that

$$
\begin{aligned}
N_{1}+N_{2} & =s^{2} t_{2}-s t_{2}-s+t_{2}+s\left(t-t_{2}\right) t+\left(t_{2}+1\right) s\left(t-t_{2}\right), \\
\sum_{R \in \mathcal{R}} t_{R}^{2} & =t(t+1)-t_{2}^{2}-\frac{n_{2}}{s^{2}} \\
n_{2} & =\frac{v}{s+1}-1+s^{2} t-s t
\end{aligned}
$$

we can compute that

$$
a_{22}=s^{2} t_{2}-2 s t_{2}-s+t_{2}+s t-t-\frac{1}{s}+\frac{v}{s(s+1)} .
$$

Observe that the latter number remains valid if $s=1$. Indeed, above we already showed that $a_{22}=\frac{v}{2}-2$ if $s=1$.

Finally, suppose that $\delta=3$. By the triangle inequality, we have $a_{00}=a_{01}=a_{02}=$ $a_{10}=a_{20}=a_{11}=0$. On each line through $x$, there is a unique point at distance 2 from $y$, implying that $a_{12}=\left|\Gamma_{1}(x) \cap \Gamma_{2}(y)\right|=t+1$. Similarly, we have that $a_{21}=t+1$. We will now compute $a_{22}$.

If $s=1$, then since $\mathcal{S}$ is a bipartite graph, we have $a_{22}=\left|\Gamma_{2}(x) \cap \Gamma_{2}(y)\right|=0$. Assume therefore that $s \geq 2$.

Let $\mathcal{R}_{1}$ denote the set of quads through $x$ containing a point of $\Gamma_{1}(y)$, and let $\mathcal{R}_{2}$ denote the set of all remaining quads through $x$. Again, $\mathcal{R}:=\mathcal{R}_{1} \cup \mathcal{R}_{2}$ is the set of all quads through $x$.

Lemma 3.5 The number of shortest paths between $x$ and $y$ is equal to $t+1+\sum_{R \in \mathcal{R}_{1}} t_{R}$.

Proof. For every point $u \in \Gamma_{2}(x) \cap \Gamma_{1}(y)$, let $u^{\prime}$ denote a common neighbour of $u$ and $x$. Then there are $t+1$ shortest paths between $x$ and $y$ of the form $x u^{\prime} u y$. For every $R \in \mathcal{R}_{1}$, the point $x$ and the unique point $u \in \Gamma_{1}(y) \cap R$ have $t_{R}$ common neighbours distinct from $u^{\prime}$, giving rise to $t_{R}$ extra shortest paths between $x$ and $y$. By Proposition 2.1, we know that we have accounted for all shortest paths between $x$ and $y$ now. So, the total number of them should be $t+1+\sum_{R \in \mathcal{R}_{1}} t_{R}$.

Lemma 3.6 We have $a_{22}=(s-1)\left((t+1)^{2}-\sum_{R \in \mathcal{R}} t_{R}^{2}\right)$.

Proof. We count in two different ways the number of pairs $(u, z)$ satisfying $z \in \Gamma_{2}(x) \cap$ $\Gamma_{2}(y)$ and $u \in \Gamma_{1}(x) \cap \Gamma_{1}(z)$.

We show that there are $(s-1)(t+1) t$ such pairs $(u, z)$ where $u \in \Gamma_{1}(x) \cap \Gamma_{3}(y)$. Indeed, there are $(s-1)(t+1)$ points $u \in \Gamma_{1}(x) \cap \Gamma_{3}(y)$, and for a given $u$ there are $t$ possibilities for $z$, namely one on each line through $u$ distinct from $u x$.

We show that there are $(s-1)\left(t+1+\sum_{R \in \mathcal{R}_{1}} t_{R}\right)$ such pairs $(u, z)$ with $u \in \Gamma_{1}(x) \cap \Gamma_{2}(y)$. If $(u, z)$ is such a pair, then the fact that $\mathrm{d}(u, y)=\mathrm{d}(z, y)=2$ implies that the line $u z$ contains a point $u^{\prime}$ collinear with $y$, implying that $x u u^{\prime} y$ is a shortest path from $x$ to $y$. Conversely, if $x u u^{\prime} y$ is a shortest path from $x$ to $y$ and $z$ is one of the $s-1$ points 
of $u u^{\prime} \backslash\left\{u, u^{\prime}\right\}$, then $(u, z)$ is a suitable pair. From Lemma 3.5, it now follows that the number of suitable pairs $(u, z)$ with $u \in \Gamma_{1}(x) \cap \Gamma_{2}(y)$ is equal to $(s-1)\left(t+1+\sum_{R \in \mathcal{R}_{1}} t_{R}\right)$.

So, the total number of suitable pairs is equal to $(s-1)\left((t+1)^{2}+\sum_{R \in \mathcal{R}_{1}} t_{R}\right)$. We now count the number of pairs $(u, z)$ in a different way.

If $R \in \mathcal{R}_{1}$, then $y$ has distance 1 from a unique point $y^{\prime}$ of $R$, implying that $y$ is classical with respect to $R$. Since $\mathrm{d}(x, y)=3$, we have $\mathrm{d}\left(x, y^{\prime}\right)=2$. The quad $R$ contains $(s-1)\left(t_{R}+1\right)$ points $z$ of $\Gamma_{2}(x) \cap \Gamma_{2}(y)$ (namely the points of $R \backslash\left\{y^{\prime}\right\}$ collinear with $y^{\prime}$, but not with $x$ ) and each of these points is collinear with $t_{R}+1$ points $u \in \Gamma_{1}(x)$.

If $R \in \mathcal{R}_{2}$, then $y$ is ovoidal with respect to $R$, and so $R$ contains $s t_{R}+1$ points of $\Gamma_{2}(y), t_{R}+1$ of which are collinear with $x$, implying that there are $(s-1) t_{R}$ points $z \in R \cap \Gamma_{2}(x) \cap \Gamma_{2}(y)$. Moreover, each such point $z$ is collinear with $t_{R}+1$ points $u \in \Gamma_{1}(x)$.

Since each of the $a_{22}-(s-1) \cdot \sum_{R \in \mathcal{R}_{1}}\left(t_{R}+1\right)-(s-1) \cdot \sum_{R \in \mathcal{R}_{2}} t_{R}$ points of $\Gamma_{2}(x) \cap \Gamma_{2}(y)$ not contained in a quad together with $x$ is collinear with a unique point $u \in \Gamma_{1}(x)$, we see that the number of suitable pairs is also equal to

$$
\begin{gathered}
a_{22}-(s-1) \sum_{R \in \mathcal{R}_{1}}\left(t_{R}+1\right)-(s-1) \sum_{R \in \mathcal{R}_{2}} t_{R}+(s-1) \sum_{R \in \mathcal{R}_{1}}\left(t_{R}+1\right)^{2} \\
+(s-1) \sum_{R \in \mathcal{R}_{2}} t_{R}\left(t_{R}+1\right)=a_{22}+(s-1) \sum_{R \in \mathcal{R}_{1}} t_{R}\left(t_{R}+1\right)+(s-1) \sum_{R \in \mathcal{R}_{2}} t_{R}^{2} .
\end{gathered}
$$

Since this number is equal to $(s-1)\left((t+1)^{2}+\sum_{R \in \mathcal{R}_{1}} t_{R}\right)$, we see that $a_{22}=(s-1)((t+$ $\left.1)^{2}-\sum_{R \in \mathcal{R}} t_{R}^{2}\right)$

Since $\sum_{R \in \mathcal{R}} t_{R}^{2}=t(t+1)-\frac{n_{2}}{s^{2}}$, we find that $a_{22}=(s-1)\left(t+1+\frac{n_{2}}{s^{2}}\right)$. If we replace in the latter expression $n_{2}$ by $\frac{v}{s+1}-1+s^{2} t-s t$, then we find

$$
a_{22}=\frac{(s-1)(2 s-1)}{s} t+\frac{\left(s^{2}-1\right)(s-1)}{s^{2}}+\frac{v(s-1)}{s^{2}(s+1)} .
$$

Observe that the latter number remains valid if $s=1$. Indeed, above we have already shown that $a_{22}=0$ if $s=1$.

We summarize the values of $a_{i j}$ in Table 1 . Recall that for $\delta=\mathrm{d}(x, y)=2$, the number $t_{2}$ need not to be constant and can depend on the points $x$ and $y$. The fact that $t_{2}$ is not necessarily constant has no influence on the validity of the arguments in Section 4.

\section{An idempotent matrix}

In this section, $\mathcal{S}=(\mathcal{P}, \mathcal{L})$ denotes a finite near hexagon of order $(s, t)$ having $v$ points. We will consider a particular ordering $p_{1}, p_{2}, \ldots, p_{v}$ of the points, and use this ordering to define real matrices $A_{i}, i \in\{0,1,2,3\}$, whose rows and columns are indexed by the points. For every $i \in\{0,1,2,3\}$ and all $x, y \in \mathcal{P}$, we define

$$
\left(A_{i}\right)_{x y}= \begin{cases}1 & \text { if } \mathrm{d}(x, y)=i \\ 0 & \text { otherwise }\end{cases}
$$




\begin{tabular}{|c||c|c|c||c||c|c|c|}
\hline$\delta=0$ & 0 & 1 & 2 & $\delta=2$ & 0 & 1 & 2 \\
\hline \hline 0 & 1 & 0 & 0 & 0 & 0 & 0 & 1 \\
\hline 1 & 0 & $s(t+1)$ & 0 & 1 & 0 & $t_{2}+1$ & $(s-1)\left(t_{2}+1\right)$ \\
\hline 2 & 0 & 0 & $\frac{v}{s+1}-1+s^{2} t-s t$ & 2 & 1 & $(s-1)\left(t_{2}+1\right)$ & $s^{2} t_{2}-2 s t_{2}-s+t_{2}+s t-t-\frac{1}{s}+\frac{v}{s(s+1)}$ \\
\hline \hline$\delta=1$ & 0 & 1 & 2 & $\delta=3$ & 0 & 1 & 2 \\
\hline \hline 0 & 0 & 1 & 0 & 0 & 0 & 0 & 0 \\
\hline 1 & 1 & $s-1$ & $s t$ & 1 & 0 & 0 & $t+1$ \\
\hline 2 & 0 & $s t$ & $(s-1) s t$ & 2 & 0 & $t+1$ & $\frac{(s-1)(2 s-1)}{s} t+\frac{\left(s^{2}-1\right)(s-1)}{s^{2}}+\frac{v(s-1)}{s^{2}(s+1)}$ \\
\hline
\end{tabular}

Table 1: The intersection numbers $a_{i j}$ for $\delta \in\{0,1,2,3\}$

Then $A_{1}$ equals the collinearity matrix $A$ of $\mathcal{S}$ (with respect to the ordering) and $A_{0}$ equals $I$, the $v \times v$ identity matrix. We also define

$$
N:=\sum_{i=0}^{3}\left(-\frac{1}{s}\right)^{i} A_{i}
$$

and

$$
\alpha:=n_{0}+\frac{n_{1}}{s^{2}}+\frac{n_{2}}{s^{4}}+\frac{n_{3}}{s^{6}}=\frac{(s+1)^{2}(s-1)\left(s^{2}+1\right)+s t(s-1)(s+1)^{2}+v}{s^{5}}>0 .
$$

In this section, we prove the following result.

Proposition 4.1 We have $N \cdot N=\alpha \cdot N$. As a consequence, $\frac{N}{\alpha}$ is an idempotent matrix.

Proof. For every point $x$ of $\mathcal{S}$, let $\chi_{x}$ be the row vector whose columns are indexed by the $v$ points of $\mathcal{S}$ (with respect to the above ordering), such that $\chi_{x}(z):=\left(-\frac{1}{s}\right)^{\mathrm{d}(x, z)}$ for every point $z$ of $\mathcal{S}$. If $x$ and $y$ are two points of $\mathcal{S}$, then the standard inner product of the two row vectors $\chi_{x}$ and $\chi_{y}$ is equal to

$$
\chi_{x} \cdot \chi_{y}=\sum_{0 \leq i, j \leq 3} a_{i j}\left(-\frac{1}{s}\right)^{i+j}
$$

where $a_{i j}=\left|\Gamma_{i}(x) \cap \Gamma_{j}(y)\right|$ for all $i, j \in\{0,1,2,3\}$. Taking into account that

$$
a_{i 3}=n_{i}-a_{i 0}-a_{i 1}-a_{i 2}, \quad a_{3 i}=n_{i}-a_{0 i}-a_{1 i}-a_{2 i}, \quad a_{33}=2 n_{3}-v+\sum_{i, j=0}^{2} a_{i j},
$$

for every $i \in\{0,1,2\}$ and making use of Proposition 2.3, we see that

$$
\begin{aligned}
\chi_{x} \cdot \chi_{y} & =\left(\sum_{i, j=0}^{2} k_{i j} a_{i j}\right)-\frac{v}{s^{6}}+\left(2 n_{0}\left(-\frac{1}{s}\right)^{3}+2 n_{1}\left(\frac{1}{s}\right)^{4}+2 n_{2}\left(-\frac{1}{s}\right)^{5}+2 n_{3}\left(\frac{1}{s}\right)^{6}\right) \\
& =-\frac{v}{s^{6}}+\sum_{i, j=0}^{2} k_{i j} a_{i j},
\end{aligned}
$$


where $k_{i j}=\left(-\frac{1}{s}\right)^{i+j}-\left(-\frac{1}{s}\right)^{i+3}-\left(-\frac{1}{s}\right)^{j+3}+\frac{1}{s^{6}}$ for all $i, j \in\{0,1,2\}$, i.e.

$$
\begin{gathered}
k_{00}=\frac{\left(s^{3}+1\right)^{2}}{s^{6}}, \quad k_{01}=k_{10}=-\frac{\left(s^{2}-1\right)\left(s^{3}+1\right)}{s^{6}}, \quad k_{02}=k_{20}=\frac{(s+1)\left(s^{3}+1\right)}{s^{6}}, \\
k_{11}=\frac{\left(s^{2}-1\right)^{2}}{s^{6}}, \quad k_{12}=k_{21}=-\frac{(s+1)\left(s^{2}-1\right)}{s^{6}}, \quad k_{22}=\frac{(s+1)^{2}}{s^{6}} .
\end{gathered}
$$

Using the contents of Table 1, it is straightforward (but somewhat tedious) to compute that $\chi_{x} \cdot \chi_{y}=\alpha \cdot\left(-\frac{1}{s}\right)^{\mathrm{d}(x, y)}$ for every two points $x$ and $y$ of $\mathcal{S}$. If $x$ is a point of $\mathcal{S}$, then the row $x$ of the matrix $N$ coincides with $\chi_{x}$. As $N$ is a symmetric matrix, we thus have that $N \cdot N^{T}=N^{2}=\alpha \cdot N$.

Remark. For a finite near $2 d$-gon of order $(s, t)$, we can define similarly as above matrices $A_{i}, i \in\{0,1, \ldots, d\}$, and put $N:=\sum_{i=0}^{d}\left(-\frac{1}{s}\right)^{i} A_{i}$. For several known examples of such near $2 d$-gons, it holds that $N^{2}=\alpha N$ for some $\alpha \in \mathbb{Q}$. The explanation of this fact seems to come from the following result.

Lemma 4.2 If there exists a polynomial $p^{*}(x) \in \mathbb{Q}[x]$ such that $N=p^{*}(A)$, then $N^{2}=$ $\alpha N$ for some $\alpha \in \mathbb{Q}$.

Proof. It can be shown, see Proposition 1 of [13], that $A N=-(t+1) N$. This implies that $N^{2}=p^{*}(A) N=p^{*}(-(t+1)) N=\alpha N$, where $\alpha=p^{*}(-(t+1)) \in \mathbb{Q}$.

For many near $2 d$-gons, e.g. those with a distance-regular collinearity graph (for other examples, see also $[13$, Section 4$])$, it can be shown that there exist polynomials $p_{i}(x) \in \mathbb{Q}[x]$ $(i \in\{0,1, \ldots, d\})$ such that $A_{i}=p_{i}(A)$, so that one can take $p^{*}(x)=\sum_{i=0}^{d}\left(-\frac{1}{s}\right)^{i} p_{i}(x)$. However, to prove that these polynomials exist seems to be a hard problem for general finite near polygons with an order. Despite the fact that the existence of these polynomials $p_{i}(x)$ was not proved here, it was still possible (for the near hexagon case) to show that $N^{2}=\alpha N$ for some $\alpha \in \mathbb{Q}$.

We also note that if there exists a polynomial $p^{*}(x) \in \mathbb{Q}[x]$ such that $N=p^{*}(A)$, then the idempotent matrix $\frac{N}{\alpha}$ with $\alpha=p^{*}(-(t+1))$ can be regarded as the projection matrix on the eigenspace of the smallest eigenvalue $-(t+1)$ of $A$.

\section{A generalization of the Haemers-Roos inequality}

We continue with the notation of Section 4. In particular, $\mathcal{S}=(\mathcal{P}, \mathcal{L})$ is a finite near hexagon of order $(s, t)$. If $X$ is a set of points of $\mathcal{S}$, then $\gamma_{X}$ denotes the characteristic vector of $X$, i.e. the row matrix whose columns are indexed by the points of $\mathcal{S}$ (respecting the earlier mentioned ordering of points) such that $\gamma_{X}(y)=1$ if $y \in X$ and $\gamma_{X}(y)=0$ if $y \in \mathcal{P} \backslash X$. If $X$ is the singleton $\{x\}$, then we denote $\gamma_{X}$ also by $\gamma_{x}$.

Consider two opposite points $x$ and $y$ of $\mathcal{S}$, and put $Z_{1}:=\Gamma_{1}(x) \cap \Gamma_{2}(y), Z_{2}:=$ $\Gamma_{2}(x) \cap \Gamma_{1}(y)$. We show that if $z_{1} \in Z_{1}$ and $z_{2} \in Z_{2}$, then $\mathrm{d}\left(z_{1}, z_{2}\right) \in\{1,3\}$. By the triangle inequality, we know that $\mathrm{d}\left(z_{1}, z_{2}\right) \in\{1,2,3\}$. If $\mathrm{d}\left(z_{1}, z_{2}\right) \leq 2$, then (NP) and the 
fact that $\mathrm{d}\left(z_{1}, y\right)=2$ implies that there exists a unique point $z_{2}^{\prime}$ on the line $y z_{2}$ collinear with $z_{1}$. This point lies at distance at most 2 from $x$ and hence coincides with $z_{2}$, implying that $\mathrm{d}\left(z_{1}, z_{2}\right)=1$.

Since the matrix $\frac{N}{\alpha}$ is idempotent (Proposition 4.1), it is positive semidefinite, implying that also $N$ is positive semidefinite (as $\alpha>0$ ). It follows that for all $X_{1}, X_{2}, X_{3}, X_{4} \in \mathbb{R}$,

$$
\left(X_{1} \gamma_{x}+X_{2} \gamma_{y}+X_{3} \gamma_{Z_{1}}+X_{4} \gamma_{Z_{2}}\right) \cdot N \cdot\left(X_{1} \gamma_{x}+X_{2} \gamma_{y}+X_{3} \gamma_{Z_{1}}+X_{4} \gamma_{Z_{2}}\right)^{T} \geq 0,
$$

i.e.,

$$
\left[\begin{array}{llll}
X_{1} & X_{2} & X_{3} & X_{4}
\end{array}\right] \cdot M \cdot\left[\begin{array}{llll}
X_{1} & X_{2} & X_{3} & X_{4}
\end{array}\right]^{T} \geq 0
$$

where

$$
M=\left[\begin{array}{cccc}
1 & -\frac{1}{s^{3}} & -\frac{t+1}{s} & \frac{t+1}{s^{2}} \\
-\frac{1}{s^{3}} & 1 & \frac{t+1}{s^{2}} & -\frac{t+1}{s} \\
-\frac{t+1}{s} & \frac{t+1}{s^{2}} & \frac{(t+1)\left(s^{2}+t\right)}{s^{2}} & \gamma \cdot\left(-\frac{1}{s}\right)+\left((t+1)^{2}-\gamma\right) \cdot\left(-\frac{1}{s^{3}}\right) \\
\frac{t+1}{s^{2}} & -\frac{t+1}{s} & \gamma \cdot\left(-\frac{1}{s}\right)+\left((t+1)^{2}-\gamma\right) \cdot\left(-\frac{1}{s^{3}}\right) & \frac{(t+1)\left(s^{2}+t\right)}{s^{2}}
\end{array}\right],
$$

with $\gamma$ denoting the number of shortest paths connecting $x$ and $y$. It follows that $M$ is also positive semidefinite matrix. By Sylvester's criterion for positive semidefinite matrices [21, Theorem 7.2.5], we then know that

$$
\operatorname{det}(M(1)) \geq 0, \quad \operatorname{det}(M(2)) \geq 0, \quad \operatorname{det}(M(3)) \geq 0, \quad \operatorname{det}(M(4)) \geq 0,
$$

where $M(i)$ is the submatrix of $M$ defined by the first $i$ rows and first $i$ columns of $M$.

If $s=1$, then $\operatorname{det}(M(1))=1$ and $\operatorname{det}(M(2))=\operatorname{det}(M(3))=\operatorname{det}(M(4))=0$, and so the inequalities for $\operatorname{det}(M(i))$ are always fulfilled.

Suppose therefore that $s \geq 2$. Then $\operatorname{det}(M(1))=1, \operatorname{det}(M(2))=1-\frac{1}{s^{6}}>0$ and

$$
\operatorname{det}(M(3))=\frac{t+1}{s^{8}}\left(s^{2}-1\right)^{2}\left(s^{4}+s^{2}-t\right),
$$

implying that

$$
t \leq s^{4}+s^{2} .
$$

If $x$ and $y$ are two points at distance 2 having at least two common neighbours, then Proposition 2.1 implies that $x$ and $y$ are contained in a unique quad of order $\left(s, t_{x y}\right)$ for some $t_{x y} \in \mathbb{N} \backslash\{0\}$. By Higman [20, (6.4)], we know that $t_{x y} \leq s^{2}$. This implies that every two opposite points of $\mathcal{S}$ are connected by at most $(t+1)\left(s^{2}+1\right)$ shortest paths, i.e.

$$
\gamma \leq(t+1)\left(s^{2}+1\right)
$$

We compute

$$
\begin{aligned}
& \operatorname{det}(M(4))=-\frac{1}{s^{12}}\left(s^{2}-1\right)^{3}\left((t+1)^{2}-\left(s^{2}+s+1\right)(\gamma-s(t+1))\right) \cdot\left((t+1)^{2}-\left(s^{2}-s+1\right)(\gamma+s(t+1))\right), \\
& =-\frac{1}{s^{12}}\left(s^{2}-1\right)^{3}(t+1)^{2} \cdot\left(t+1-\left(s^{2}+s+1\right)(\bar{\gamma}-s)\right) \cdot\left(t+1-\left(s^{2}-s+1\right)(\bar{\gamma}+s)\right),
\end{aligned}
$$


where $\bar{\gamma}:=\frac{\gamma}{t+1} \leq s^{2}+1$. The latter inequality implies that $\left(s^{2}+s+1\right)(\bar{\gamma}-s) \leq$ $\left(s^{2}-s+1\right)(\bar{\gamma}+s)$. The fact that $\operatorname{det}(M(4)) \geq 0$ then implies that

$$
\left(s^{2}+s+1\right)(\bar{\gamma}-s) \leq t+1 \leq\left(s^{2}-s+1\right)(\bar{\gamma}+s)
$$

If we put $\overline{t_{2}}:=\bar{\gamma}-1$, then we find

$$
\left(s^{2}+s+1\right) \overline{t_{2}}-s^{3} \leq t \leq s^{3}+\overline{t_{2}}\left(s^{2}-s+1\right) .
$$

As mentioned earlier, the upper bound for $t$ generalizes the Haemers-Roos inequality for generalized polygons [19] and the Haemers-Mathon bound for regular near hexagons $[18,24,26]$. The lower bound for $t$ generalizes a lower bound obtained in [15]. In fact, the techniques we invoked in this section are similar to some of the techniques used in [15] and [32].

We will now deal with the case where $t$ attains the upper bound $s^{4}+s^{2}$ (under the assumption that $s>1$ ). If $t=s^{4}+s^{2}$, then (1) implies that $\overline{t_{2}}=s^{2}$, i.e. $\bar{\gamma}=s^{2}+1$ and $\gamma=(t+1)\left(s^{2}+1\right)$. By the above we then know that every two points at distance 2 have precisely $s^{2}+1$ common neighbours. This implies that every two points at distance 2 are contained in a unique quad of order $\left(s, s^{2}\right)$, or equivalently, that every two intersecting lines are contained in a unique quad of order $\left(s, s^{2}\right)$. For every point $x$, consider now the point-line geometry $\mathcal{S}_{x}$ with point set $\mathcal{L}_{x}=\{L \in \mathcal{L} \mid x \in L\}$ and line set $\mathcal{L}_{x}:=$ $\left\{L_{Q} \mid Q\right.$ a quad through $\left.x\right\}$, where $L_{Q}$ is the set consisting of all lines through $x$ contained in $Q$. The point-line geometry $\mathcal{S}_{x}$ satisfies the following properties:

- there are $s^{4}+s^{2}+1$ points and every line contains $s^{2}+1$ points;

- every two distinct points are incident with a unique line.

So, $\mathcal{S}_{x}$ is a Steiner system $S\left(2, s^{2}+1, s^{4}+s^{2}+1\right)$ and hence a projective plane of order $s^{2}$. As this holds for all points $x$ of $\mathcal{S}$, the near hexagon $\mathcal{S}$ must be a dual polar space by Shult and Yanushka [29, Lemma 2.21]. By the classification of polar spaces (Veldkamp [33], Tits [31]), we then know that $\mathcal{S}$ must be a Hermitian dual polar space, i.e. $s$ is a prime power and $\mathcal{S}$ is isomorphic to $\operatorname{DH}\left(5, s^{2}\right)$.

\section{Application to tetrahedrally closed line systems}

Every tetrahedron $T$ centered at the origin $o$ of the Euclidean 3 -space $\mathbb{R}^{3}$ determines a tetrahedrally closed system $\Sigma_{T}$ of norm 3 vectors of type $\left(0, \frac{1}{3}\right)$ for which all four local near polygons are lines of size 3. There are up to isomorphism three generalized quadrangles of order $(2, *)$ (namely, the $(3 \times 3)$-grid, $W(2)$ and $Q(5,2))$, and by Shult and Yanushka [29] or Cuypers [10] we already know that each of them occurs as a local near polygon of a suitable tetrahedrally closed system of norm 3 vectors of type $\left(0, \frac{1}{3}\right)$. So, it suffices to prove Theorem 1.2 in the case of near hexagons.

Suppose $\mathcal{S}=(\mathcal{P}, \mathcal{L})$ is a finite near hexagon with three points per line and denote by $\mathrm{d}(\cdot, \cdot)$ the distance function in $\mathcal{S}$. Put $\mathcal{P}=\left\{p_{1}, p_{2}, \ldots, p_{v}\right\}$ with $v=|\mathcal{P}|$ and denote by $\infty$ 
a symbol that is not contained in $\mathcal{P}$. Similarly as in Section 4 , we can define real $v \times v$ matrices $A_{0}, A_{1}, A_{2}, A_{3}, N$ with respect to the ordering $\left(p_{1}, p_{2}, \ldots, p_{v}\right)$ of the points. We now define an additional matrix $M$ which has dimensions $(v+1) \times(v+1)$ and whose rows and columns are indexed by the set $\{\infty\} \cup \mathcal{P}$ using the ordering $\left(\infty, p_{1}, p_{2}, \ldots, p_{v}\right)$. The matrix $M$ is defined as follows:

- $M_{\infty, \infty}:=3$;

- $M_{\infty, x}:=-1$ and $M_{x, \infty}:=-1$ for every point $x \in \mathcal{P}$;

- $M_{x, x}:=3$ if $x \in \mathcal{P}, M_{x, y}:=-1$ if $x, y \in \mathcal{P}$ with $\mathrm{d}(x, y)=1, M_{x, y}:=1$ if $x, y \in \mathcal{P}$ with $\mathrm{d}(x, y)=2$, and $M_{x, y}:=0$ if $x, y \in \mathcal{P}$ with $\mathrm{d}(x, y)=3$.

Let $P$ denote the following real $(v+1) \times(v+1)$-matrix:

$$
P:=\left(\begin{array}{cccccc}
1 & \frac{1}{3} & \frac{1}{3} & \cdots & \frac{1}{3} & \frac{1}{3} \\
0 & 1 & 0 & \cdots & 0 & 0 \\
0 & 0 & 1 & \cdots & 0 & 0 \\
\vdots & \vdots & \vdots & \ddots & \vdots & \vdots \\
0 & 0 & 0 & \cdots & 1 & 0 \\
0 & 0 & 0 & \cdots & 0 & 1
\end{array}\right) .
$$

It is straightforward to verify that $P^{T} \cdot M \cdot P$ is the following block matrix:

$$
\left(\begin{array}{cc}
3 & 0 \\
0 & \frac{8}{3} \cdot N
\end{array}\right)
$$

So, the matrix $M$ is positive-semidefinite if and only if $N$ is positive semidefinite.

Proposition 6.1 The following are equivalent:

(1) $\mathcal{S}$ is some local near polygon of a tetrahedrally closed system of norm 3 vectors of type $\left(0, \frac{1}{3}\right)$.

(2) The matrix $N$ is positive semidefinite.

Proof. We use some of the notation introduced in Proposition 1.1. Suppose $\mathcal{S}=\mathcal{S}_{\Sigma, \bar{v}}$, where $\Sigma$ is a tetrahedrally closed system of norm 3 vectors of type $\left(0, \frac{1}{3}\right)$ of $\mathbb{R}^{n}$ satisfying Property $\left(P_{\bar{v}}\right)$ where $\bar{v}$ is some vector of $\Sigma$. By Proposition 1.1, we then know that $M$ is a Gram matrix of the set $\{\bar{v}\} \cup \mathcal{P}_{\Sigma, \bar{v}}$, implying (see e.g. [21, Section 7.2]) that $M$ and hence also $N$ are positive semidefinite.

Conversely, suppose that $N$ and hence also $M$ are positive semidefinite matrices. If $n$ is the rank of $M$, then by [21, Section 7.2], there exists a collection $\Sigma$ of vectors in $\mathbb{R}^{n}$ for which $M$ is a Gram matrix.

Denote by $\bar{v} \in \Sigma$ the vector corresponding to the first row and column of $M$. Every vector of $\Sigma \backslash\{\bar{v}\}$ then corresponds to a point of $\mathcal{S}$ which we will denote by $\theta(\bar{v})$. We define

$$
\bar{\Sigma}:=\Sigma \cup-\Sigma,
$$


where $-\Sigma:=\{-\bar{u} \mid \bar{u} \in \Sigma\}$. Since all diagonal entries of $M$ are equal to 3 and all nondiagonal entries are equal to $-1,1$ or 0 , we see that $\Sigma \cap(-\Sigma)=\emptyset$ and $\bar{\Sigma}$ is system of norm 3 vectors of type $\left(0, \frac{1}{3}\right)$. Since all non-diagonal elements in the first row and column of $M$ are equal to -1 , we see that $\mathcal{P}_{\bar{\Sigma}, \bar{v}}=\{\bar{u} \in \bar{\Sigma} \mid \bar{v} \cdot \bar{u}=-1\}=\Sigma \backslash\{\bar{v}\}$.

The remainder of the proof can now be completed as in Proposition 3.14 of the paper [29] by Shult and Yanushka. In particular, it now follows from [29] that $\bar{\Sigma}$ is tetrahedrally closed and satisfies Property $\left(P_{\bar{v}}\right)$. The map $\theta$ moreover defines an isomorphism between the point-line geometries $\mathcal{S}_{\bar{\Sigma}, \bar{v}}$ and $\mathcal{S}$.

Theorem 1.2 of Section 1 is an immediate consequence of Propositions 4.1 and 6.1, taking into account that any idempotent matrix is also positive semidefinite.

\section{References}

[1] M. Aschbacher. Flag structures on Tits geometries. Geom. Dedicata 14 (1983), 21-32.

[2] A. Bishnoi and B. De Bruyn. A new near octagon and the Suzuki tower. Electron. J. Combin. 23 (2016), Paper 2.35, 24 pp.

[3] A. E. Brouwer, A. M. Cohen and A. Neumaier. Distance-regular graphs. SpringerVerlag, 1989.

[4] P. J. Cameron. Dual polar spaces. Geom. Dedicata 12 (1982), 75-85.

[5] P. J. Cameron, J.-M. Goethals, J. J. Seidel and E. E. Shult. Line graphs, root systems, and elliptic geometry. J. Algebra 43 (1976), 305-327.

[6] A. M. Cohen. Geometries originating from certain distance-regular graphs. pp. 8187 in "Finite geometries and designs" (Proc. Conf., Chelwood Gate, 1980). London Math. Soc. Lecture Note Ser. 49, Cambridge Univ. Press, 1981.

[7] A. M. Cohen and J. Tits. On generalized hexagons and a near octagon whose lines have three points. European J. Combin. 6 (1985), 13-27.

[8] J. H. Conway and N. J. A. Sloane. Sphere packings, lattices and groups. Third edition. Grundlehren der Mathematischen Wissenschaften 290. Springer-Verlag, 1999.

[9] H. S. M. Coxeter. Twelve points in PG $(5,3)$ with 95040 self-transformations. Proc. Roy. Soc. London Ser. A 247 (1958), 279-293.

[10] H. Cuypers. Extended near hexagons and line systems. Adv. Geom. 4 (2004), 181214.

[11] B. De Bruyn. Near hexagons with four points on a line. Adv. Geom. 1 (2001), 211-228.

[12] B. De Bruyn. Bounding the size of near hexagons with lines of size 3. J. Graph Theory 52 (2006), 108-122.

[13] B. De Bruyn. On the adjacency algebras of near hexagons with an order. Graphs Combin. 33 (2017), 1219-1230.

[14] B. De Bruyn and M. Gao. The classification of a certain caps in finite projective spaces. In preparation. 
[15] B. De Bruyn and F. Vanhove. Inequalities for regular near polygons, with applications to $m$-ovoids. European J. Combin. 34 (2013), 522-538.

[16] G. Greaves, J. H. Koolen, A. Munemasa and F. Szöllösi. Equiangular lines in Euclidean spaces. J. Combin. Theory Ser. A 138 (2016), 208-235.

[17] J. Haantjes. Equilateral point-sets in elliptic two- and three-dimensional spaces. Nieuw Arch. Wiskunde 22 (1948), 355-362.

[18] W. Haemers and R. Mathon. An inequality for near hexagons. Unpublished manuscript (1979).

[19] W. Haemers and C. Roos. An inequality for generalized hexagons. Geom. Dedicata 10 (1981), 219-222.

[20] D. G. Higman. Partial geometries, generalized quadrangles and strongly regular graphs. pp. 263-293 in Atti del Convegno di Geometria Combinatoria e sue Applicazioni (Univ. Perugia, Perugia, 1970). Ist. Mat., Univ. Perugia, Perugia, 1971.

[21] R. A. Horn and C. R. Johnson. Matrix analysis. Second edition. Cambridge University Press, Cambridge, 2013.

[22] J. Leech. Notes on sphere packings. Canad. J. Math. 19 (1967), 251-267.

[23] J. H. van Lint and J. J. Seidel. Equilateral point sets in elliptic geometry. Indag. Math. 28 (1966), 335-348.

[24] R. Mathon. On primitive association schemes with three classes. Unpublished manuscript.

[25] A. Neumaier. On norm three vectors in integral Euclidean lattices. I. Math. Z. 183 (1983), 565-574.

[26] A. Neumaier. Krein conditions and near polygons. J. Combin. Theory Ser. A 54 (1990), 201-209.

[27] J. J. Seidel. Graphs and two-graphs. pp. 125-143 in "Proceedings of the Fifth Southeastern Conference on Combinatorics, Graph Theory and Computing" (Florida Atlantic Univ., Boca Raton, Fla., 1974). Congressus Numerantium X, Utilitas Math., 1974.

[28] J. J. Seidel. Discrete non-Euclidean geometry. pp. 843-920 in Handbook of incidence geometry. North-Holland, 1995.

[29] E. E. Shult and A. Yanushka. Near n-gons and line systems. Geom. Dedicata 9 (1980), $1-72$.

[30] J. Tits. Sur la trialité et certains groupes qui s'en déduisent. Inst. Hautes Etudes Sci. Publ. Math. 2 (1959), 13-60.

[31] J. Tits. Buildings of spherical type and finite BN-pairs. Lecture Notes in Mathematics 386. Springer-Verlag, 1974.

[32] F. Vanhove. A Higman inequality for regular near polygons. J. Algebraic Combin. 34 (2011), 357-373.

[33] F. D. Veldkamp. Polar Geometry. I-IV, V. Indag. Math. 21 and 22 (1959), 512-551 and 207-212. 